University of Nebraska - Lincoln

DigitalCommons@University of Nebraska - Lincoln

$5-1-2001$

\title{
A Surface Insulator-to-Conductor Phase Transition in Colossal Magnetoresistive Manganese Perovskites Thin Films
}

\author{
C.N. Borca \\ University of Nebraska-Lincoln \\ Bo Xu \\ University of Nebraska-Lincoln \\ Takashi Komesu \\ University of Nebraska-Lincoln, tkomesu2@unl.edu \\ Hae-Kyung Jeong \\ University of Nebraska-Lincoln, hjeong@unl.edu \\ Sy-Hwang Liou \\ University of Nebraska-Lincoln, sliou@unl.edu \\ See next page for additional authors
}

Follow this and additional works at: https://digitalcommons.unl.edu/physicsdowben

Part of the Physics Commons

Borca, C.N.; Xu, Bo; Komesu, Takashi; Jeong, Hae-Kyung; Liou, Sy-Hwang; and Dowben, Peter A., "A Surface Insulator-to-Conductor Phase Transition in Colossal Magnetoresistive Manganese Perovskites Thin Films" (2001). Peter Dowben Publications. 168.

https://digitalcommons.unl.edu/physicsdowben/168

This Article is brought to you for free and open access by the Research Papers in Physics and Astronomy at DigitalCommons@University of Nebraska - Lincoln. It has been accepted for inclusion in Peter Dowben Publications by an authorized administrator of DigitalCommons@University of Nebraska - Lincoln. 


\section{Authors}

C.N. Borca, Bo Xu, Takashi Komesu, Hae-Kyung Jeong, Sy-Hwang Liou, and Peter A. Dowben 


\title{
A Surface Insulator-to-Conductor Phase Transition in Colossal Magnetoresistive Manganese Perovskites Thin Films
}

\author{
C.N. Borca, Bo Xu, Takashi Komesu, Hae-Kyung Jeong, S.-H. Liou, P.A. Dowben \\ Department of Physics and Astronomy and the Center for Material Research and Analysis, \\ University of Nebraska, Lincoln, NE 68588-0111, U.S.A.
}

\begin{abstract}
We have observed a distinct surface phase transition for an important class of colossal magnetoresistive materials, $\mathrm{La}_{0.65} \mathrm{D}_{0.35} \mathrm{MnO}_{3}$ (with $\mathrm{D}=\mathrm{Sr}, \mathrm{Pb}$ ) occurring in a surface layer compositionally different from the bulk. The surface phase transition occurs around $240 \mathrm{~K}$ compared to $350 \mathrm{~K}$ for the bulk and is fundamentally different. In the bulk, a ferromagnetic metal to paramagnetic 'bad metal' occurs, while the lower-temperature surface transition is from an $n$-type (in case of $\mathrm{La}_{0.65} \mathrm{Sr}_{0.35} \mathrm{MnO}_{3}$ ) or a $p$-type (in case of $\mathrm{La}_{0.65} \mathrm{~Pb}_{0.35} \mathrm{MnO}_{3}$ ) semiconductor to a semimetal with increasing temperature.
\end{abstract}

\section{INTRODUCTION}

Surface phase transitions and surface electronic structure can have a great impact on the electronic transport properties through the surface region. For materials (like manganese perovskites) considered as candidates for spin valves and spin tunnel junctions, the effects may be profound.

Phase transitions, largely restricted to the surface, have been observed in ferroelectric [1] and ferromagnetic [2] materials, and across a nonmetal to metal transition [3]. Surface transitions for complex oxides must be also considered. We report on a novel surface phase transition for the manganese perovskite $\mathrm{La}_{0.65} \mathrm{D}_{0.35} \mathrm{MnO}_{3}$ (with $\mathrm{D}=\mathrm{Sr}, \mathrm{Pb}$ ) that occurs at a different temperature than the bulk ferromagnetic phase transition.

Tailoring surfaces of complex CMR compounds may lead to improvements in device performances. The current results shed considerable light on the device performance of the CMR perovskites in tunnel magneto-resistive junctions and spin valves in the thin film limit.

\section{EXPERIMENTAL DETAILS}

The $\mathrm{La}_{0.65} \mathrm{Sr}_{0.35} \mathrm{MnO}_{3}$ (LSMO) and $\mathrm{La}_{0.65} \mathrm{~Pb}_{0.35} \mathrm{MnO}_{3}$ (LPMO) thin films (1000 $\AA$ in thickness) were grown on (100) $\mathrm{LaAlO}_{3}$ substrates by rf sputtering [4]. The films are polycrystalline, highly oriented along the substrate normal and single phase. Detailed studies of the surface composition and electronic structure were performed into an ultra high vacuum (UHV) system, with a base pressure of $2 \times 10^{-10}$ torr. To insure surface cleanness, we annealed the samples up to $520^{\circ} \mathrm{C}$ for 2 hours before each experiment. A clear four-fold symmetric low energy electron diffraction (LEED) pattern, together with a negligible amount of $\mathrm{C}$ (less than $3 \%$ of a monolayer) monitored using X-ray photoemission (XPS), were taken as good indicators of a clean surface with a high degree of crystallinity across the entire area of the sample. It has been shown that this thermal treatment completely changes the surface and selvedge structure in both 
$\mathrm{La}_{0.65} \mathrm{Sr}_{0.35} \mathrm{MnO}_{3}$ [5] and $\mathrm{La}_{0.65} \mathrm{~Pb}_{0.35} \mathrm{MnO}_{3}$ [6] thin films. Compare to the well-known bulk structure [7], these annealed surfaces go through a restructuring transition characterized by the formation of a Ruddlesden-Popper phase $\left(\mathrm{La}_{1-\mathrm{x}} \mathrm{D}_{\mathrm{x}}\right)_{2} \mathrm{MnO}_{4}(\mathrm{D}=\mathrm{Sr}, \mathrm{Pb})$ with a possibly embedded $\mathrm{MnO}$ columnar phase.

The influence of surface compositions on the conductivity in the surface region can be observed in temperature dependence of core level binding energies as well as in temperature dependence of unoccupied levels lying above the Fermi threshold. We use x-ray photoemission spectroscopy (XPS) to monitor the binding energy shifts of the oxygen core level as a function of temperature. The XPS measurements were undertaken with the $\mathrm{Mg} \mathrm{K}_{\alpha}$ line $(1253.6 \mathrm{eV})$ using a commercial system. The temperature dependence of the unoccupied levels above the Fermi energy has been explored using inverse photoemission spectroscopy. In this technique, incident electrons with very low kinetic energies impinge the sample surface and decay to unoccupied levels by emitting a photon. In our measurements we sweep the energy of the incident electrons and, at the same time, we monitor the monochromatic photons using an in-house build Geiger Mueller detector.

\section{DISCUSSION}

Figure 1 shows the temperature dependence of the O-1s core level at different X-ray source powers. Panel (a) corresponds to the O- $1 s$ in LPMO thin films, while panel (b) presents the O-1s core level shifts in LSMO. As can be seen, the trend of the core level shift is similar for both types of surfaces. The greater the incident x-ray flux becomes, the larger the core level shift - toward higher binding energies - for temperatures below $240 \mathrm{~K}$. The difference between the two surfaces consists in the amount of the core-level shift, which is approximately $2.5 \mathrm{eV}$ for LSMO and $1.25 \mathrm{eV}$ for LPMO surface at, for example, $200 \mathrm{~W}$ source power.

In order to understand this temperature dependent behavior, we have to take into account the screening effects on the oxygen $1 s$ core level. In the final state of the photoemission process, the core photoelectron has enough energy to exit the surface, leaving behind a core-hole. An
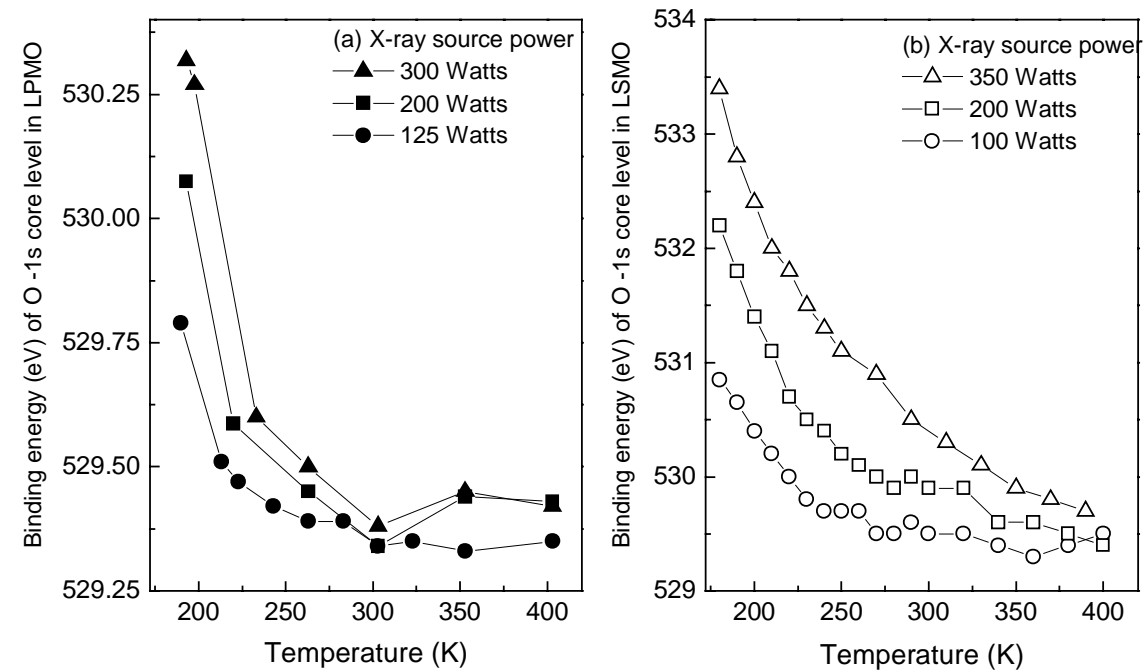

Figure 1. The binding energy of the oxygen $1 s$ peak as a function of temperature and x-ray power for (a) $\mathrm{La}_{0.65} \mathrm{~Pb}_{0.35} \mathrm{MnO}_{3}$ and (b) $\mathrm{La}_{0.65} \mathrm{Sr}_{0.35} \mathrm{MnO}_{3}$ thin films. 
increase in binding energy of the outgoing electron is equivalent to a decrease of its kinetic energy. If the outgoing electron is well screened from the photo-hole, the kinetic energy will increase. In the opposite case, when the photo-hole is poorly screened, the kinetic energy of the electron in the final state will decrease. Therefore, the shift of the binding energy with temperature provides a direct indicator of the more metallic (good screening) or insulator (poor screening) character of the surface region. By changing the x-ray power, we change the number of photo-holes created in the surface region and the core level shift should scale proportionally with the number of the core-holes existent in the final state.

As mentioned above, the $\mathrm{O} 1 \mathrm{~s}$ core level exhibits a bigger core level shift with increasing $\mathrm{x}$-ray flux in the case of LSMO compare to LPMO, at temperatures below $240 \mathrm{~K}$. This indicates that the screening of the core-hole in case of LSMO is much weaker than in the case of LPMO, which makes the surface of LSMO more insulating than the LPMO surface.

The reason for this unexpected surface insulator-like behavior at low temperature is the surface restructuring induced by the heavy annealing treatments in both materials. The Ruddlesden-Popper phase ( $n=1$ stacking sequence) created at the surface of LSMO [5] might extend deeper in the selvedge region than in the case of LPMO [6]. Note that the bulk structures of both CMR materials are identical, even though the doping element is different, corresponding to $n=\infty$ stacking sequence.

The nature of the insulator - at different temperatures - can be additionally probed using inverse photoemission spectroscopy. Figure 2 shows the temperature dependent spectra acquired

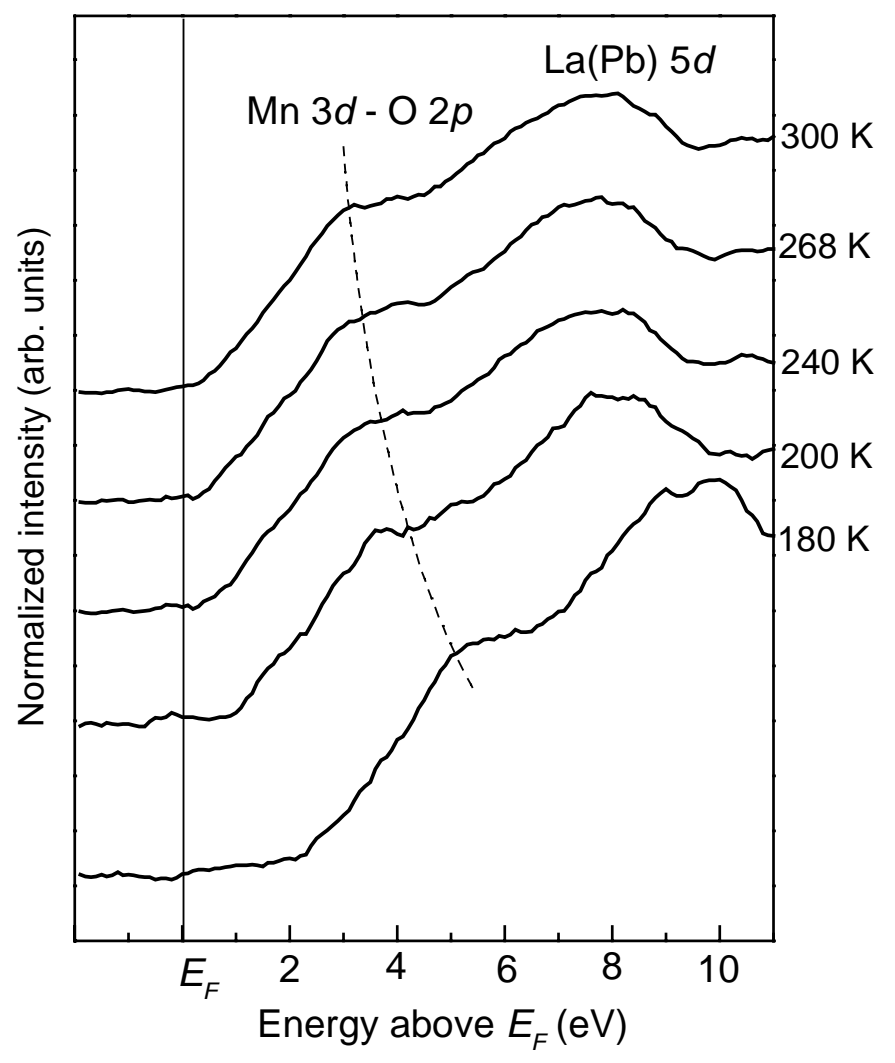

Figure 2. Inverse p hotoemission spectra acquired at normal emission as a function of temperature for the restructured $\mathrm{La}_{0.65} \mathrm{~Pb}_{0.35} \mathrm{MnO}_{3}$ surface. Fermi level is indicated with a straight vertical line. 
at normal incidence from the LPMO surface. A similar temperature behavior was obtained from the LSMO surface in reference [5]. In both $\mathrm{Sr}$ - and $\mathrm{Pb}$-doped perovskite structures, the dramatic decrease in density of states at $\mathrm{E}_{\mathrm{F}}$ with lowering temperature indicates that the surfaces become insulating, consistent with the O-1s core level shift shown in Figure 1. Two broad unoccupied bands can be identified al all temperatures in Figure 1. The band present at high energy above $\mathrm{E}_{\mathrm{F}}$, from approximately 5 to $8 \mathrm{eV}$ is mostly due to the $\mathrm{La}(\mathrm{Pb}) 5 d$ band, while the shoulder structure centered around $3.6 \mathrm{eV}$ above $\mathrm{E}_{\mathrm{F}}$ originates from the nonbonding or antibonding-like states with Mn $3 d-\mathrm{O} 2 p$ hybridized character $[8,9]$.

In comparison with other CMR-manganite materials, the Mn $3 d-\mathrm{O} 2 p$ antibonding-like states are positioned at approximately $2 \mathrm{eV}$ above the Fermi level for both $\mathrm{La}_{0.65} \mathrm{Ca}_{0.35} \mathrm{MnO}_{3}$ and $\mathrm{La}_{0.65} \mathrm{Ba}_{0.35} \mathrm{MnO}_{3}$, while the same states are found at about $4 \mathrm{eV}$ above Fermi level for the $\mathrm{La}_{0.65} \mathrm{Sr}_{0.35} \mathrm{MnO}_{3}[10]$. The feature at $6.5 \mathrm{eV}$, corresponding to the La $5 d$ states, is clearly observed in the conduction band of $\mathrm{La}_{0.65} \mathrm{Ca}_{0.35} \mathrm{MnO}_{3}$, while the $\mathrm{La} 5 d$ state for $\mathrm{La}_{0.65} \mathrm{Sr}_{0.35} \mathrm{MnO}_{3}$ are substantially broader and less apparent, as in case of $\mathrm{La}_{0.65} \mathrm{Ba}_{0.35} \mathrm{MnO}_{3}$. In general, the similarity of the spectra for all doped manganites argues in favor of the model proposed by Taguchi and Shimada [11] for $\mathrm{La}_{1-\mathrm{x}} \mathrm{Ca}_{\mathrm{x}} \mathrm{MnO}_{2.97}$, proving that the bonding is generally covalent and not ionic for the moderately doped manganite species.

Combined photoemission and inverse photoemission studies performed on the LPMO surface as a function of temperature clearly show that the restructured surface resembles a $p$-type semiconductor at temperatures below $240 \mathrm{~K}$, as the unoccupied levels bend away from the Fermi level and the valence band structures remain fixed in energy with lowering temperature [12]. Less clear is the case of the LSMO restructured surface, where only temperature dependent conduction band studies are available at this time [5], which indicate an $n$-type behavior below $240 \mathrm{~K}$. It is important to stress that even though the two materials have very similar bulk magnetic and transport properties, the enthalpy difference between the surface and the bulk induces different types of charging of the insulating surface at low temperatures. It has been also proven that, as the perovskite thin films become thinner and thinner, surface properties can have an increasingly greater influence on magnetization and devices [13]. We now attribute these effects to a surface layer with different composition and electronic structure.

\section{CONCLUSIONS}

In conclusion, we have shown strong evidence for a novel surface phase transition (at around $240 \mathrm{~K}$ ) in thin films of Sr-doped and $\mathrm{Pb}$-doped manganese perovskites, which occurs at a different temperature than the bulk ferromagnetic ordering transition (of $350 \mathrm{~K}$ ). In the surface region of both materials, below $240 \mathrm{~K}$, there is a substantial shift in the $(1 s)$ core levels of oxygen, as well as in the unoccupied band structure. The surface charging effects, which induce this temperature behavior using different spectroscopies, are more pronounced in the restructured surface of LSMO than in the case of LPMO surface. This difference may be due to the presence of a Ruddlesden-Popper phase which extends deeper in the selvedge region of LSMO than in case of LPMO. The magnetic bulk measurements do not show any indication of a phase transition in this temperature region, which suggests that the surface is not intrinsically ferromagnetic. The surface of these perovskite materials is electronically and compositionally different from the bulk with important implications for the behavior of the artificially grown layered structures. 


\section{REFERENCES}

1. J. Choi et al., Phys. Rev. Lett. 80, 1328 (1998)

2. B. Gubank, M. Donath and F. Passek, Phys. Rev. B 54, R11153 (1994); D. Li, M. Freitag, J. Pearson, Z.-Q. Qui and S.D. Bader, Phys. Rev. Lett. 72, 3112 (1994); J. Thomasson, F. May, B. Feldman, M. Wuttig and H. Ibach, Phys. Rev. Lett. 69, 3831 (1992)

3. Luca Gavioli, Maria Grazia Betti and Carlo Mariani, Phys. Rev. Lett. 77, 3869 (1990); S.D. Kevan and N.G. Stoffel, Phys. Rev. Lett. 53, 702 (1984)

4. Q.L. Xu, M.T. Liu, Y.Liu, C.N. Borca, H. Dulli, P.A. Dowben, S.-H. Liou, Mat. Res. Soc. Symp. Proc. 602 (2000), in press

5. H. Dulli, E.W. Plummer, Jaewu Choi, S.-H. Liou, P.A. Dowben, Appl. Phys. Lett. 77, 570 (2000)

6. C.N. Borca, Bo Xu, Takashi Komesu, Hae-Kyung Jeong, M.T. Liu, S.-H. Liou, P.A. Dowben, submitted to Appl. Phys. Lett.

7. C.N. Borca, S. Adenwalla, S.-H. Liou, Q.L. Xu, J.L. Robertson, P.A. Dowben, submitted to J. Magn. Magn. Mat.; S.J. Hibble et al., J. Phys. Cond. Matter 11, 9221 (1999)

8. Y.Kuwata, S. Suga, S. Imada, A. Sekiyama, S. Ueda, T. Iwasaki, H. Harada, T. Muro, T. Fukawa, K. Ashida, H. Yoshioka, T. Terauchi, J. Sameshima, H. Kuwahara, Y. Morimoto, Y. Tokura, J. El. Spectros. Rel. Phenom. 88-91, 281 (1998)

9. S. Suga, et al., J. Electron Spectros. Rel. Phenom. 78, 283 (1996)

10. Jaewu Choi, H. Dulli, S.-H. Liou, P.A. Dowben, M.A. Langell, Phys. Stat. Sol. (b) 214, 45 (1999)

11. H. Taguchi, M. Shimada, J. Solid State Chem. 67, 37 (1987)

12. C.N. Borca, Bo Xu, Takashi Komesu, Hae-Kyung Jeong, M.T. Liu, S.-H. Liou, S. Stadler, Y. Idzerda, P.A. Dowben, submitted to Phys. Rev. Lett.

13. J.Z. Sun, D.W. Abraham, R.A. Rao and C.B. Eom, Appl. Phys. Lett. 74, 3017 (1999) 